\title{
Down-regulation of Id1 and Id3 genes affects growth and survival of Human Umbilical Vein Endothelial Cells (HUVECs)
}

\author{
Foster Kyei $^{1 *}$, Du-Bois Asante ${ }^{2}$, Juliet Ama Mawusi Edekor ${ }^{1}$, Esther Sarpong ${ }^{1}$, Edem Gavor ${ }^{1}$, Daniels Konja ${ }^{2}$ \\ ${ }^{1}$ College of Agriculture and Natural Sciences, School of Biological Sciences, Department of Molecular Biology and Biotechnology, University of Cape \\ Coast, Cape Coast, Ghana. ${ }^{2}$ College of Agriculture and Natural Sciences, School of Biological Sciences, Department of Biomedical and Forensic Sciences, \\ University of Cape Coast, Ghana.
}

\section{ARTICLE INFO}

Article history:

Received on: $14 / 12 / 2015$

Revised on: $15 / 01 / 2016$

Accepted on: 22/02/2016

Available online: 21/04/2016

Key words:

Apoptosis, Angiogenesis, Sphase, Down-regulation,

Human umbilical vein endothelial cells, Knockdown

\begin{abstract}
Inhibitors of DNA (Id) proteins have been implicated in cell growth, cell cycle control, apoptosis and angiogenesis. Studies reveal that Id1 and Id3 may be required for angiogenesis in adult tissues. This work aimed at investigating the consequences of loss-of-function of Id protein on growth and apoptosis in an in vitro model using Human Umbilical Vein Endothelial Cells (HUVECs). Using automated image analysis software (CellProfiler Analyst), cell cycle profiles of cell populations following siRNA down-regulation of Id proteins were investigated. Also, cell viability analysis with 3-(4, 5-Dimethylthiazol-2-yl)-2, 5-diphenyltetrazolium bromide (MTT) assay was evaluated. The study also employed flow cytometry using mitochondrial membranepotential marker (3, 3'-Dihexyloxacarbocyanine Iodide) (DiOC6) and Propidium Iodide (PI) as a marker of nonviable necrotic cells. Down-regulation of Id1 and Id3 reduced viable HUVECs populations. In addition, knock down of Id1 and Id3 showed cell cycle arrest. Furthermore, cell death analysis following the silencing of Id1 and Id3 induced apoptosis. These findings suggest that the inhibition of Id1 and Id 3 may impair endothelial cell activation and angiogenesis and may also provide an attractive target for the development of new therapies for angiogenic disorders.
\end{abstract}

\section{INTRODUCTION}

Helix-Loop-Helix (HLH) proteins are one of the important classes of transcription factors involved in the coordinate regulation of gene expression which regulate cell differentiation and lineage commitment in most organisms [1]. Accumulating evidence has implicated Id proteins in their involvement in the regulation of molecular networks and cellular processes. Notable among these are cell growth, differentiation, senescence, apoptosis, angiogenesis and neoplastic transformation [2]. The family of Helix-Loop-Helix (HLH) proteins could be identified as having over 200 members [3]. A subfamily of the HLH proteins known as Id proteins (Inhibitor of differentiation) has no DNA binding domain. The biological functions of Id proteins are achieved by associating with the ubiquitous E proteins and thereby preventing them from binding

* Corresponding Author

College of Agriculture and Natural Sciences, School of Biological

Sciences, Department of Molecular Biology and Biotechnology,

University of Cape Coast, Ghana. Email: fkyei[at]ucc.edu.gh
DNA or other transcription factors. Four members of the Id family of proteins have been identified in vertebrates namely; Id1, Id2, Id3 and Id4. Id function is involved in growth and angiogenesis of tumour cells and its regulation at intracellular levels with smallmolecule-based inhibitors of these proteins might result in the development of useful drugs to treat human cancers [4]. Id proteins are widely known to be produced by tumour cells and tumour endothelium but cannot be identified with normal adult tissues. These findings have raised the hopes that the Id proteins could be a target for cancer therapy. Many recent in vivo studies have revealed that Id proteins are potential candidates as targets for cancer therapy [5]. The application of anti-sense and small interference siRNA-based techniques has shown that the two major phenotypic hallmarks of tumour aggressiveness, invasion and metastasis are disrupted when Id protein levels in tumour cells are forcibly lowered. Overexpression of Id1 in human umbilical vascular endothelial cells (HUVECs) activated the expression of $\beta 1$-integrin and promoted cell growth, adhesion and spreading. Conversely, down-regulation of Id 1 suppressed $\beta 1$-integrin expression and inhibited tubulogenesis. 
HUVECs over-expressing Id1 were able to promote capillary tube formation through cytoskeleton reorganization and cell contraction [6]. Down-regulation of Id1 and Id3 with RNA interference abolished proliferation, activation, and angiogenic processes of HUVECs [7]. These observations indicate that Id proteins play a crucial role in endothelial cells by causing activation and potentiation of angiogenic mechanisms. Many tumour types exhibit elevated levels of at least one Id mRNA and much emphasis is being placed on the analysis of protein expression in primary tumours [8].

It has been established that angiogenesis is critically involved in the process of chronic inflammation and joint destruction in rheumatoid arthritis [9]. Recent evidence suggests that Id 1 and Id 3 play pivotal roles in regulating developmental and postnatal angiogenesis [10]. Complete loss of Id1 and Id3 genes resulted in vascular malformation in the forebrain thereby causing brain hemorrhage [11]. With partial loss of Id1 and Id3 genes, neoangiogenesis is blocked in tumor xenografts and spontaneous tumors [12].

Ectopic expression of Id1 in endothelial cells enhanced angiogenicity in both in vitro and in vivo angiogenesis models [11] and however the molecular mechanism underlying the function and control of Ids in malignant transformation and angiogenesis remains virtually unknown.

It has also been observed that Id expression in tumour cell lines results in the progression of cell growth and it is more likely to associate Id proteins with tumorigenesis mechanisms [4]. A number of unsuspected roles of Id proteins have recently been disclosed. $\mathrm{Id} 1^{+/-} \mathrm{Id} 3^{-/-}$mice were unable to support angiogenesis necessary for the progression of tumor xenografts [10], suggesting that Id 1 and Id 3 may also be required for the angiogenesis in adult tissues. Here, role of Id in angiogenesis in an in vitro model using Human Umbilical Vein Endothelial cells (HUVECs) is studied. This work investigated whether the suppression of Id proteins could inhibit the activation and angiogenic processes in the Human Umbilical Vein Endothelial Cells. Hence, this work aimed at investigating the consequences of loss-of-function of Id protein on growth and pro-apoptotic functions in HUVECs using siRNA gene knock-down. This objective was achieved by using antisense oligonucleotides complementary to Id1, Id 2 and Id 3 mRNA on HUVECs.

\section{MATERIALS AND METHODS}

\subsection{Endothelial cell culture and Id silencing}

Human Umbilical Vein Endothelial cells (HUVECs) (American Type Culture Collection, USA) were cultured in large vessel endothelial basal medium (TCS Cellworks, Buckingham) enriched with $10 \%$ large vessel endothelial cell growth supplement (TCS Cellworks, Buckingham). Cells were cultured in T25 and T75 flasks. During passaging, stocks of cells were washed in $2 \mathrm{ml}$ of sterile $1 \times \mathrm{PBS}$ and trypsinised with $1 \mathrm{ml}$ of $1 \times$ Trypsin (prewarmed) (trypsin from GIBCO, and trypsin-EDTA from PAA Laboratories, Austria) and incubated in $5 \% \mathrm{CO}_{2}$ incubator at $37^{\circ} \mathrm{C}$
(Sanyo, Japan) for 5 minutes. Thereafter $6 \mathrm{ml}$ of complete large endothelial basal medium containing $1 \%$ Gentamicin (PAA Laboratories, Austria) was added to the flask of cells to inactivate the action of the trypsin. Cells were then plated in fresh flasks, labelled (name and date) and returned into the incubator. Cells were passaged every 3 days and cultured in 5\% $\mathrm{CO}_{2}$ incubator to maintain the viability of the adherent cells. Cells were also monitored daily for evidence of contamination.

The cells were propagated at $2 \times 10^{4} /$ well in $0.5 \mathrm{ml}$ of incomplete medium. After $4 \mathrm{~h}$ of seeding, $75 \mu \mathrm{l}$ of DEPC (Diethylpyrocarbonate)-PBS was added to $9 \mu$ l of Hiperfect reagent (QIAGEN, Netherlands) and mixed gently. Again $2.25 \mu \mathrm{l}$ of Id-siRNA (QIAGEN, Netherlands) (20 $\mu \mathrm{M}$ stock) was added to the DEPC-PBS/Hiperfect-mix and the whole mixture was gently mixed again.

The mixture was incubated at room temperature for 10 min and approximately $25 \mu \mathrm{l}$ of the whole mixture was added drop-wise to each replicate well (making a final concentration of the Id siRNA to be $30 \mathrm{nM}$ ). The plate was returned into the $5 \%$ $\mathrm{CO}_{2}$ incubator at $37^{\circ} \mathrm{C}$. During the siRNA transfections, the Id siRNAs were incubated on ice and both RNAse free Eppendorf tubes and Gilson tips were used throughout the transfections. Id1, Id 2 and Id 3 were then transiently silenced by gene specific siRNA (Table 1).

Table 1: siRNA duplex Oligoribonucleotides for Id1, Id2 and Id3 proteins.

\begin{tabular}{ccc}
\hline $\begin{array}{c}\text { Id } \\
\text { Protein }\end{array}$ & Primer Name & \multicolumn{1}{c}{ RNA } \\
\hline Id1 & ID1HSS142592 & GCA AGG UGA GCA AGG UGG AGA UUC U \\
& ID1HSS142592 & AGA AUC UCC ACC UUG CUC ACC UUG C \\
\hline \multirow{2}{*}{ Id2 } & ID2HSS105176 & ACG UCA UCG ACU ACA UCU UGG ACC U \\
& ID2HSS105176 & AGG UCC AAG AUG UAG UCG AUG ACG U \\
\hline \multirow{2}{*}{ Id3 } & ID3HSS142595 & AGA AUC UCC ACC UUG CUC ACC UUG C \\
& ID3HSS142595 & GGC ACU CAG CUU AGC CAG GUG GAA A \\
\hline
\end{tabular}

Transfection efficiency was examined by BX-41 fluorescence microscopy (Olympus, Tokyo). Approximately, $8 \times 10^{4} /$ well of HUVECs was propagated in 12-well plates and transfected with 30nM fluorescent siRNA (QIAGEN, Netherlands) with Hiperfect reagent (QIAGEN, Netherlands) and Hoechststained thereafter at $24 \mathrm{~h}$ post-transfection to estimate the transfection efficiency. The efficiency of fluorescent siRNA transfection after $24 \mathrm{~h}$ shows approximately $100 \%$.

\subsection{Cell proliferation assay}

MTT (3-(4, 5-Dimethylthiazol-2-yl)-2, 5diphenyltetrazolium bromide) assay (Sigma-Aldrich, USA) was used to evaluate the proliferative activity of HUVECs. In a 24-well plate, $25 \mu \mathrm{l}$ of the MTT working stock $(10 \mathrm{mg} / \mathrm{ml})$ was added directly onto cells grown in $0.5 \mathrm{ml}$ of complete growth medium. The methodology followed is elaborated in earlier study [13].

\subsection{Cell cycle assay}

DNA content of cells (integrated intensity) using Hoechst dye counter stained with EdU (5-ethynyl-2-deoxyuridine) was 
determined. The EdU imaging kit ("Click-It EdU”, Invitrogen, USA) was used to detect the cycling HUVECs. The DNA contented analysis was performed as outlined previously [13]. To confirm the results obtained with the Hoechst-EdU regime, Propidium Iodide (PI) (Sigma-Aldrich, USA) was also tested as described in earlier study [13]. Data from the flow cytometer were analyzed using WinMDI 2.9 software. Sub-populations of cells were gated and quantified from the profiles generated.

\subsection{Cell death assay/analysis}

In quantifying apoptotic, necrotic and viable populations of HUVECs, DiOC6 (3, 3'-dihexyloxacarbocyanine iodide) (Invitrogen, USA) was counter stained with PI as previously described [13]. Data from the flow cytometer were analysed using WinMDI 2.9 software. Sub-populations of cells were gated and quantified from the density plots generated.

\subsection{SDS-PAGE Protein Electrophoresis and Western blot analysis}

HUVECs lysates were prepared for SDS-PAGE analysis followed by a West blot as described earlier [13].

\subsection{Statistical analysis}

Statistical analysis was carried out using online available tool (http://faculty.vassar.edu/lowry/VassarStats.html). The probability $(p)$ values were then calculated for the significance of the difference between the test and control proportions. Also student $t$-test was used to compare the significance of the difference between controls and test samples. Differences were considered to be statistically significant at $p<0.05(*)$, highly significant at $p<0.001(* *)$, very highly significant at $p<0.0001$ $(* * *)$.

\section{RESULTS}

\subsection{Knock-down of Id1 and Id3 Reduces the Population of Viable HUVECs but not Id2}

Viability of HUVECs was investigated by siRNA downregulation of Id1, Id 2 and Id 3 proteins compared with mock transfectants and control. Here $2 \times 10^{4} /$ well of HUVECs cells was propagated in 24-well plates and transfected with Hiperfect reagent. MTT assay was then performed on the cells at $72 \mathrm{~h}$ postransfection.

The absorbance values were then measured spectrophotometrically. By using MTT as a measure of viability, Id1 and Id3 down regulation showed a significant reduction in the population of viable HUVECs. However, knock-down of Id2 resulted in no significant reduction in viable populations of HUVECs (Fig. 1).

Protein levels of Id1, Id 2 and Id 3 following knock-down was compared to endogenous levels by western blot (Fig. 1). The results show that Id 2 is not detected in HUVECs. However, downregulation of $\mathrm{Id} 1$ and $\mathrm{Id} 3$ resulted in reduction in levels of these proteins efficiently.

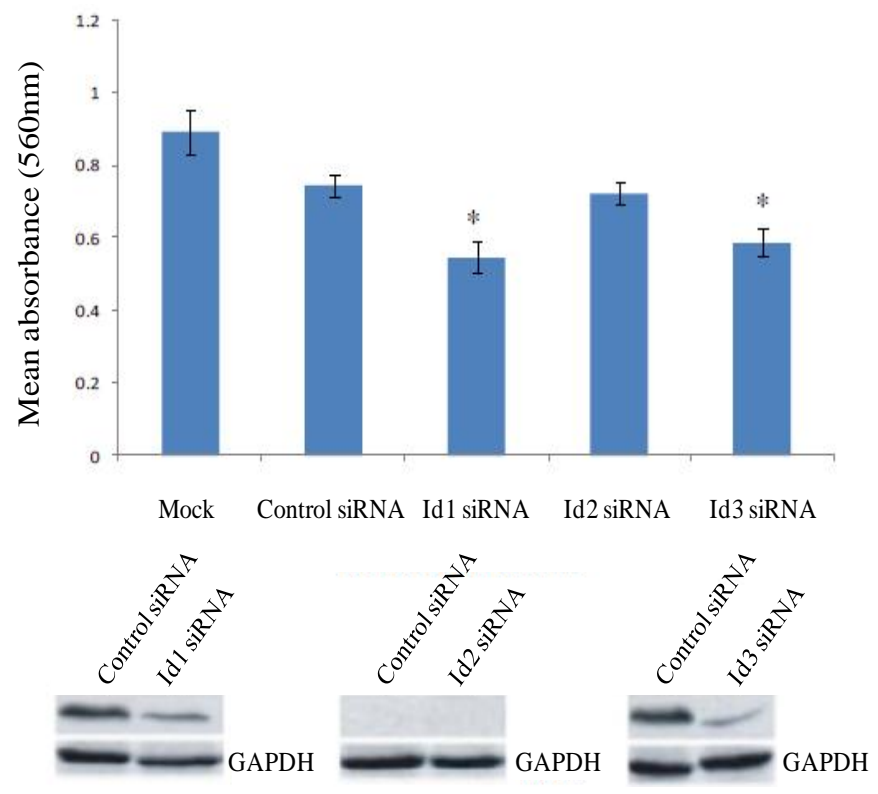

Fig. 1: Cell viability analysis of HUVECs transfected with control siRNA (30 $\mathrm{nM})$ or Id 1 siRNA $(30 \mathrm{nM})$, Id2 siRNA $(30 \mathrm{nM})$ and Id 3 siRNA (30 nM) using Hiperfect reagent after $72 \mathrm{~h}$. A: Bar graph showing relative mean absorbance following siRNA knock-down of Id1-3. Knock-down of Id1 and Id3 significantly reduced the population of viable HUVECs. Mean absorbance \pm S.E.M of 4 separate/independent samples for each transfection. B: Western blot analysis showing endogenous and siRNA down-regulated levels of Id1-3 proteins. GAPDH was used as loading control.

\subsection{Silencing of Id1 and Id3 Suppresses Growth of HUVECs}

Following earlier observation implicating Id1 and Id3 to reduce viable populations of HUVECs upon siRNA downregulation, it was necessary to investigate if this finding is as a result of suppression of growth. Here, $8 \times 104 /$ well of HUVECs were plated in 6-well plates and transfected with control siRNA, Id 1 siRNA, Id 3 siRNA and Id 3 siRNA combined with Id 1 siRNA using Hiperfect reagent and processed for EdU S-phase detection after $24 \mathrm{~h}$. Cells were processed into siliconised $1.5 \mathrm{ml}$ Eppendorf tubes and stained with Hoechst dye for DNA content analysis. After pulsing with EdU, the cells were processed onto saline coated microscope slides for digital image acquisition. After running the cells through CellProfiler software, CellProfiler Analyst was used to generate scatter plots for estimation of cell cycle parameters. It was observed that siRNA down-regulation of Id1 and Id3 in HUVECs significantly suppresses S-phase cells. Also double knock- down of both Id 1 and Id 3 resulted in growth arrest (Fig. 2).

In validation of the above results, cell cycle analysis by staining cells with PI was carried out on the HUVECs. $8 \times 104 /$ well of cells was plated in 6-well plates and transfected with control siRNA, Id1 siRNA, Id2 siRNA and Id3 siRNA using Hiperfect reagent. At 24 and $48 \mathrm{~h}$ post-transfection, both viable and nonviable cells were harvested and fixed followed by treatment with RNAse. Lastly cells were stained with PI and processed through the flow. 

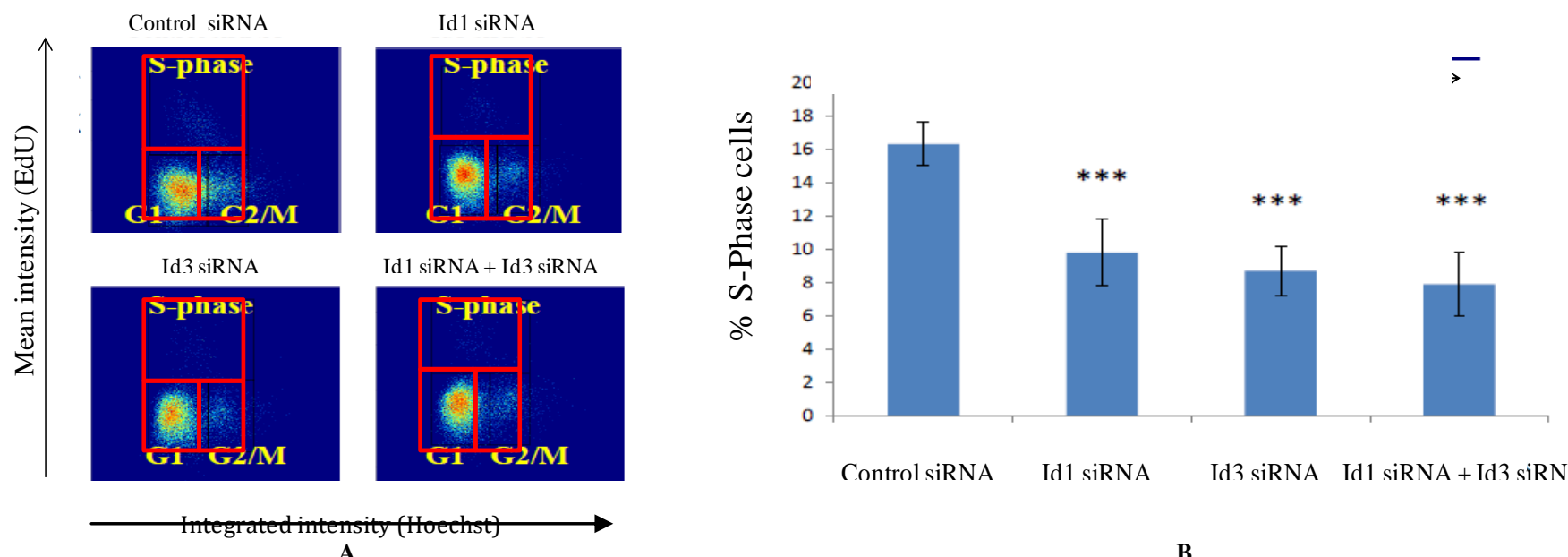

Control siRNA Id1 siRNA Id 3 siRNA Id 1 siRNA + Id 3 siRNA

A

Fig. 2: Cell cycle profiles generated using CellProfiler Analyst and percentage cell cycle parameters in HUVECs after knocking down of Id genes using Hiperfect after $24 \mathrm{~h}$. Cells were stained with EdU for S-phase detection and counter stained with Hoechst dye for 45 min. Digital images were taken and processed through CellProfiler for automated image analysis. A: Cell cycle profiles from control siRNA (30 nM), siRNA Id1 (30 nM), siRNA Id3 (30 nM) and double knock-downs with siRNA Id1 and siRNA Id3 at $24 \mathrm{~h}$ post-transfection. B: Percentage S-phase cells at 24 h post-transfection. Means \pm S.E.M of 3 separate independent experiments.

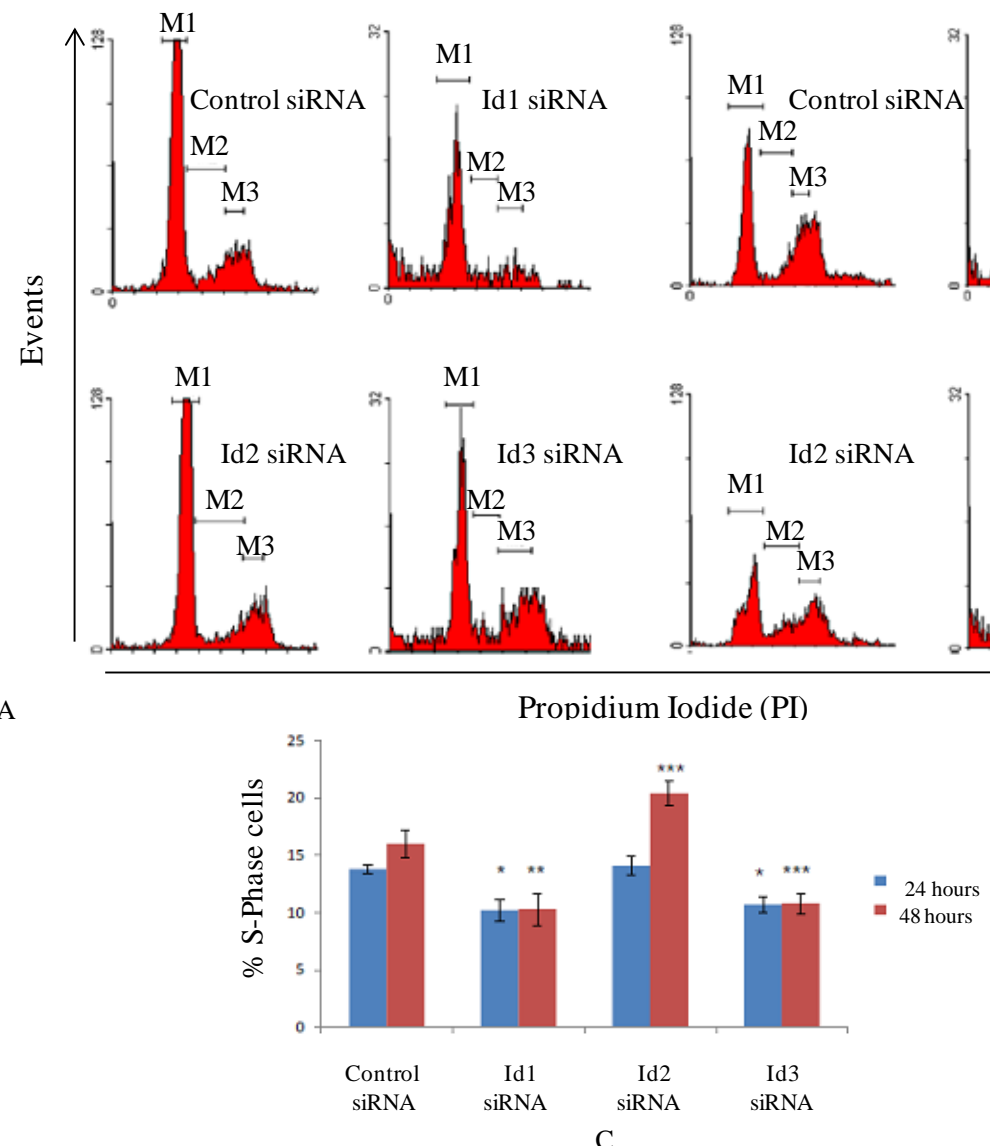

Fig. 3: Flow cytometric analysis of HUVECs showing cell cycle profiles from PI (50 $\mu \mathrm{g} / \mathrm{ml})$ staining of cells upon knock-down of Id1 (30 $\mathrm{nM})$, Id2 ( $30 \mathrm{nM})$,

The results showed a significant reduction on proliferation following knock down of Id 1 and Id 3 proteins at 24 and $48 \mathrm{~h}$ post-transfection. Down-regulation of Id2 in HUVECs however did not result in growth arrest (Fig. 3). These results are in agreement with what was previously observed with EdU Sphase analysis and confirm the effects of down-regulation of Id1, Id 2 and Id 3 on growth of HUVECs. Further to these observations, the results also showed widespread cell death induced by knocking down of Id 1 and Id 3 as represented by the sub-G1 population (representing both apoptotic and necrotic cells) of the cell cycle profiles obtained from the PI staining. Here it was also evident that down-regulation of Id1 and Id3 in HUVECs induce cell death at both 24 and $48 \mathrm{~h}$ post-transfection but not Id2 (Fig. 4). 


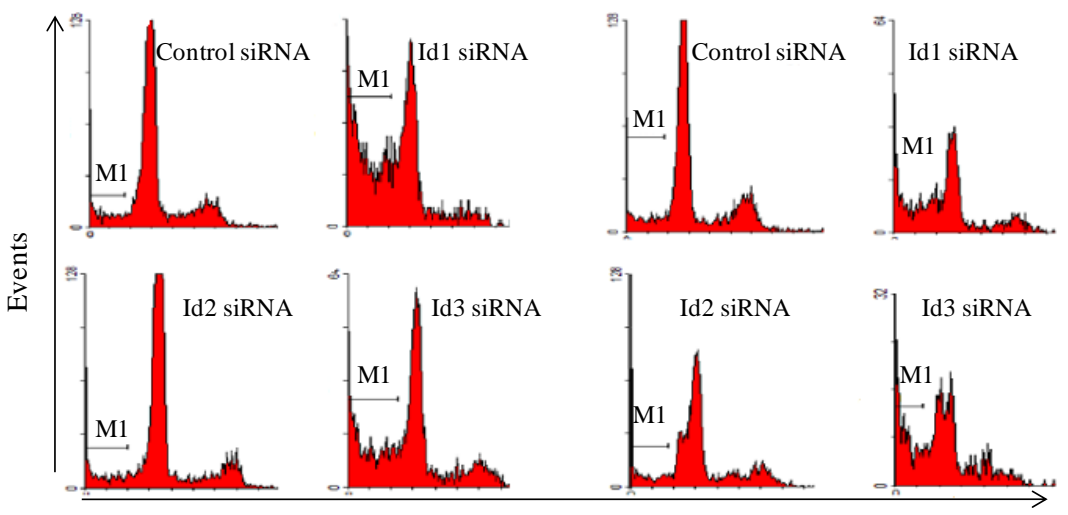

A

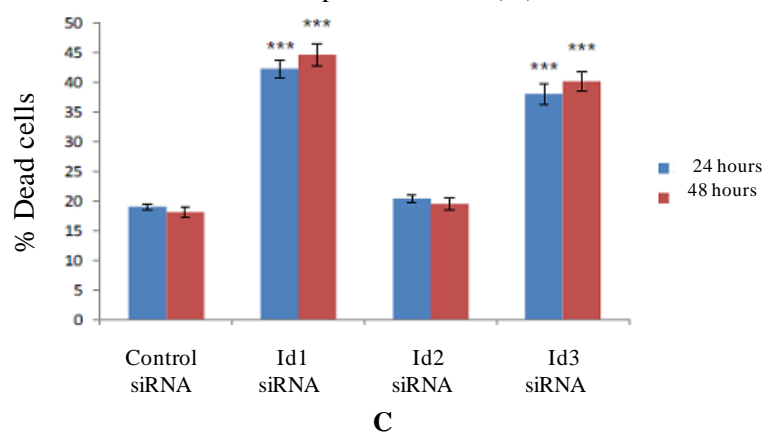

B

Fig. 4: Flow cytometric analysis of HUVECs showing cell cycle profiles from PI (50 $\mu \mathrm{g} / \mathrm{ml})$ staining of cells upon knock-down of Id1, Id2, and Id3 proteins. A: Cell cycle profiles showing sub-G1 cell populations at $24 \mathrm{~h}$ post-transfection. B: Cell cycle profiles showing sub-G1 populations after 48 hours. C: Percentage dead cell populations at 24 and $48 \mathrm{~h}$ post-transfection. Means \pm S.E.M of 3 separate independent experiments. $30 \mathrm{nM}$ siRNA was used for each transfection.
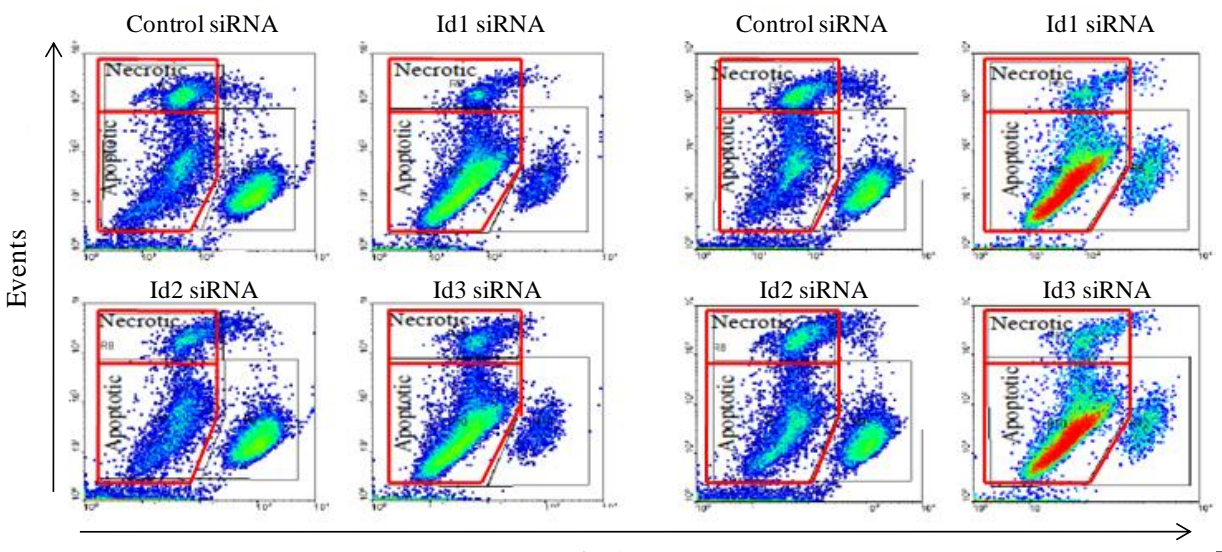

A

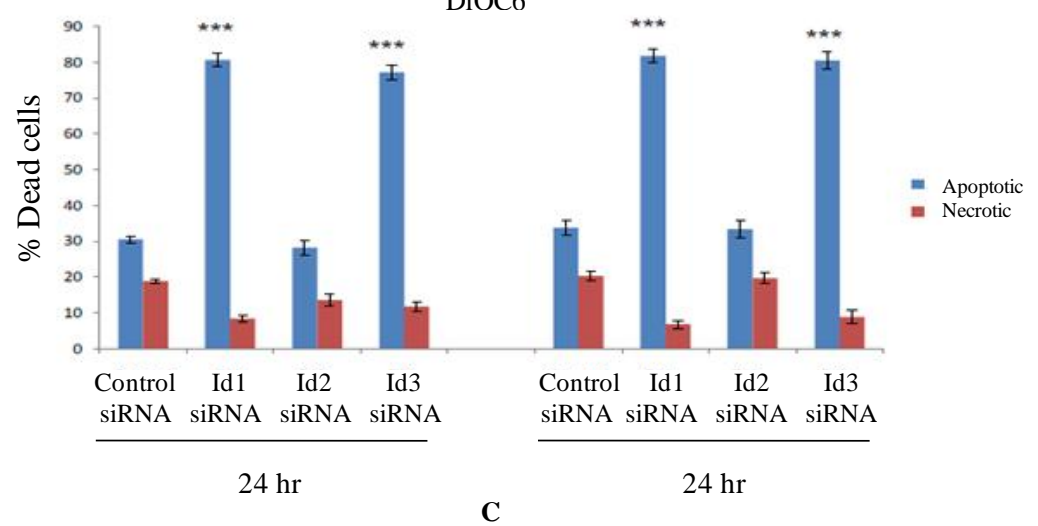

Fig. 5: Flow cytometric analysis of HUVECs for viable, apoptotic and necrotic cell population using DiOC6 and PI following knock down of Id1, Id2 and Id3. A: Gated apoptotic and necrotic populations from control siRNA, Id1 siRNA, Id2 siRNA, and Id3 siRNA after $24 \mathrm{~h}$. B: Apoptotic and necrotic populations from cells transfected with control siRNA, Id1 siRNA, Id2 siRNA, and Id3 siRNA at $48 \mathrm{~h}$ post-transfection. C: Percentage dead cell populations after Id knock-down after 24 and $48 \mathrm{~h}$. Means \pm S.E.M of 3 separate independent experiments. 


\subsection{Down-regulation of Id1 and Id3 Induces Apoptosis in HUVECs but not Id2}

Further to the growth suppressive response and induction of widespread death observed earlier following down-regulation of Id1 and Id3, an independent apoptotic marker DiOC6, was again used on the HUVECs to validate the apoptotic effects upon knockdown of Id1 and Id3. $8 \times 104 /$ well of HUVECs was seeded in 6well plates and transfected with control siRNA, siRNA Id1, siRNA Id2 and siRNA Id3 with Hiperfect reagent. After 24 and 48 $\mathrm{h}$ of transfection, cells were stained with $50 \mathrm{nM}$ DiOC6 and counter stained with PI at $2.5 \mathrm{mg} / \mathrm{ml}$. The results showed that antisense Oligonucleotide blockade of Id1 and Id3 in HUVECs significantly induced apoptosis (Fig. 5). However knock-down of Id2 did not induce apoptosis in HUVECs but rather shows a protective effect. These observations are in agreement with earlier observations in the sub-G1 populations produced by each Id siRNA (Fig. 4), hence validates the fact that Id1 and Id3 downregulation induces apoptosis in HUVECs.

\section{DISCUSSION}

The consequences of loss-of-function of Id protein on growth and pro-apoptotic functions in Umbilical Vein Endothelial Cells (HUVECs) by siRNA gene knock down were investigated to provide a more physiologically relevant data. There is a growing body of evidence supporting the role of Id proteins in tumourigenesis and angiogenic mechanisms. Using $\mathrm{Id} 1^{+/-} \mathrm{Id} 3^{-/-}$ mice, it has been shown that Id expression is required to support angiogenesis in tumors [10]. In the present study, down-regulation of Id 1 and Id 3 in HUVECs resulted in reduction of viable cell populations (Fig. 1).

By using EdU and PI staining procedures, cell cycle analysis following down-regulation of $\operatorname{Id} 1, \operatorname{Id} 2$ and $\operatorname{Id} 3$ showed cell cycle arrest in the Id1 and Id3 knock down cells by suppressing growth. However, antisense Oligonucleotide blockade of Id 2 did not suppress S-phase cells (Fig. 2 and 3). Furthermore, cell death analysis of Id1 siRNA, Id2 siRNA and Id3 siRNA transfectants resulted in induction of apoptosis following knock down of Id1 and Id3 by both methods used (thus DiOC6 and subG1 from PI staining). These effects of down-regulation of Id1 and Id3 were noticeable as the levels of these proteins were efficiently reduced after western blot analysis (Fig. 1). However, downregulation of Id 2 did not show evidence of cell death induction (Fig. 4 and 5). Endogenous Id2 (Fig. 1) was not detected in HUVECs; hence no effects on cell death and proliferation was observed following down-regulation. These results suggest that the reduction in viable populations of HUVECs upon Id1 and Id3 down-regulation is mediated through cell cycle arrest and apoptosis. These observations also support the argument that apoptosis induction by Id proteins is complex in different cell types [14].

Evidence available suggests that inappropriate entry into S-phase of the cell cycle is associated with apoptosis suggesting that these two cellular activities are coordinately regulated [15,
16]. The application of anti-sense and small interference siRNAbased techniques has shown that the two major phenotypic hallmarks of tumour aggressiveness, invasion and metastasis are disrupted when Id protein levels in tumour cells are forcibly lowered. Thus, the reduction in protein levels of both Id1 and Id 3 in gastric cancer prevented metastatic dissemination to the peritoneum whilst Id1 during its inactivation in breast cancer cells inhibited invasion and metastasis to the lung. Also anti-sense inhibition of Id1 protein expression prevented invasiveness of breakdown cluster region (BCR)-Abelson Leukemia viral oncogene (ABL) driven to many organs $[17,18]$.

Research shows that many genes have products that regulate cell cycle progression and also play a pivotal role in the modulation of apoptosis. This finding supports a model in which activation of the cell death pathway is due to a conflict between growth-promoting and growth-inhibitory signals [19]. Id proteins control cell cycle by regulating the transcription of several target genes and also by direct association with non-bHLH proteins. The cell cycle is controlled by two different pathways during the midlate G1 phase. The first involves a direct association between the retinoblastoma proteins (pRB), Id2 and Id4. This leads to the progression of the $S$ phase by attenuating $\mathrm{pRB}$ suppression of $\mathrm{E} 2 \mathrm{~F}$ DPI transcription factors thereby driving expression of genes required for the $\mathrm{S}$-phase progression. The second pathway involves the inhibition of E2A bHLH regulated expression of the gene encoding the cyclin dependent kinase (CDK) inhibitor $\mathrm{p} 21^{\mathrm{Cip} 1} /{ }^{\text {Waf } 1}$ and these results in $\mathrm{pRB}$ phosphorylation. Id1 inhibits the expression of $\mathrm{p} 21^{\mathrm{Cip} 1} /{ }^{\text {Waf1 }}$ which is an inhibitor of the cyclindependent kinases and the phosphorylation of pRB causes its dissociation from E2F-DPI complexes into S-phase [20]. Recent investigations have however demonstrated that dual regulators of proliferation and apoptosis promote these processes by a separate mechanism [16]. Reports also implicate p53 the tumour suppressor protein, which can induce growth arrest as well as apoptosis are genetically separable [21]. The above findings imply that whilst regulators of cell death and growth pathways are shared and coordinated, their functional activities in these pathways are distinct [22].

Overexpression of Id1 in human umbilical vascular endothelial cells (HUVECs) activated the expression of $\beta 1$ integrin and promoted cell growth, adhesion and spreading. Conversely, down-regulation of Id1 suppressed $\beta 1$-integrin expression and inhibited tubulogenesis. HUVECs overexpressing Id1 were able to promote capillary tube formation through cytoskeleton reorganization and cell contraction [6]. Literature available shows that enforced over-expression of Id1 and Id3 induce proliferative activity in HUVECs accompanied by downregulation of p16INK4a. Also over-expression of Id promoted the expression of ICAM-1 and E-selectin, and induced angiogenic processes of transmigration, matrix metalloproteinase-2 and -9 expression, and tube formation [7]. Evidence also shows that silencing of Id1 and Id3 with RNA interference abolished proliferation, activation, and angiogenic processes of HUVECs [7]. These observations indicate that Id proteins play a crucial role 
in endothelial cells by causing activation and potentiation of angiogenic mechanisms. These findings suggest that Id proteins may be involved in multiple pathways that affect activation of proliferation of endothelial cells and angiogenesis.

Literature available suggests that functions of Id1 and Id3 are overlapping $[23,24]$. However it is intriguing to delineate the pathways from each Id protein to a multitude of endothelial cell activation and angiogenic processes [7]. It has also been shown that over-expression of Id1 and Id3 resulted in activation of proliferation of HUVECs but concluded that their downstream pathways may not be identical [7]. The requirement of Id proteins in human endothelial cells, up-regulation of ICAM-1 and Eselectin by forced expression of Id, and inhibition of endothelial cell activation and angiogenesis by double knock down of Id1 and Id3 further confirms the crucial role of Id proteins in endothelial cell activation and angiogenesis [7].

\section{CONCLUSION}

It has been demonstrated that Id proteins may be involved in the modulation of endothelial cell angiogenesis. Given these findings, the inhibition of expression of functions of Id1 and Id3 may potentially be of therapeutic value for conditions associated with pathological angiogenesis. Hence, the inhibition of endothelial cell activation and angiogenesis following siRNA down-regulation of Id 1 and Id 3 may provide a potential target for the development of new therapeutic approaches for angiogenic disorders.

\section{ACKNOWLEDGEMENTS}

The author acknowledges the contribution made by the gene regulation laboratory, and the Molecular Medicine group of the University of Essex.

\section{REFERENCES}

1. Norton JD. Id helix-loop-helix proteins in cell growth, differentiation and tumourigenesis. Journal of Cell Science. 2000; 113:3897-3905.

2. Hasmat A, Skider HS, Meghann K. Id proteins in cell growth and tumourigenesis. Cancer Cell. 2003; 6:525-530.

3. Massari ME, Murre C. Helix-loop-helix proteins: regulators of transcription in eukaryotic organisms. Molecular Cellular Biology. 2000; 20:429-440.

4. Lin CQ, Singh J, Murata K, Itahana Y, Parrinello S, Liang SH, et al. A role for Id1 in the aggressive phenotype and steroid hormone response of human breast cancer cells. Cancer Research. 2000; 60:1332-1340.

5. Iavarone A, Lasorella A. Id proteins as targets in cancer and tools in neurobiology. Trends in Molecular Medicine. 2006; 12:588-594.

6. Qiu J, Wang G, Peng Q, Hu J, Luo X, Zheng Y, et al. Id1 induces tubulogenesis by regulating endothelial cell adhesion and cytoskeletal organization through $\beta 1$-integrin and Rho-kinase signalling. Cancer Letters. 2011; 307:191-199.

7. Sakurai D, Tsuchiya N, Yamaguchi A, Okaji Y, Tsuno NH, Kobata $\mathrm{T}$, et al. Crucial Role of inhibitor of DNA binding/differentiation in the vascular endothelial growth factor induced activation and angiogenic processes of human endothelial cells. Journal of Immunology. 2004; 173:5801-5809.
8. Israel MA, Hernandez MC, Florio M, Andres-Barquin P, Mantani A, Carter JH, et al. Id gene expression as a key mediator of tumour cell biology. Cancer Research. 1999; 59:1726-1730.

9. Koch AE. Angiogenesis: implications for rheumatoid arthritis. Arthritis and Rheumatology. 1998; 41:951-962.

10. Lyden D, Young AZ, Zagzag D, Yan W, Gerald W, O'Reilly R, et al. Id1 and Id3 are required for neurogenesis, angiogenesis and vascularization of tumour xenografts. Nature. 1999; 40:670-677.

11. Nishiyama K, Takaji K, Kataoka K, Kurihara Y, Yoshimura M, Kato A, et al. Id 1 gene transfer confers angiogenic property on fully differentiated endothelial cells and contributes to therapeutic angiogenesis. Circulation. 2005; 112:2840-2850.

12. Ruzinova MB, Schoer RA, Gerald W, Egan JE, Pandolfi PP, Rafii S, et al. Effect of angiogenesis inhibition by Id loss and the contribution of bone marrow derived endothelial precuresor cells in spontaneous murine tumors. Cancer Cell. 2003; 4:277-289.

13. Kyei F. Knock-down of Id 1 and Id3 proteins induces apoptosis in human colon carcinoma (HCT116) cells. Journal of Biology and Life Science. 2015; 6(1):194-208.

14. Hara E, Zebedee Z. Id proteins in cell cycle control and cellular senescence. Oncogene. 2001; 20:8317-8325.

15. Askew DS, Ashmun RA, Simmons BC, Clevaland JL. Constitutive cmyc expression in an IL-3-dependent myeloid cell line suppresses cell cycle arrest and accelerates apoptosis. Oncogene. 1991; 6:19151922

16. Wagner AJ, Kokontis MJ, Hay N. Myc-mediated apoptosis requires wild-type p53 in a manner independent of cell cycle arrest and the ability of $\mathrm{p} 53$ to induce $\mathrm{p} 21^{\text {Cip1 }} /$ Waf1. Genes and Development. 1994; 8:2817-2830.

17. Fong S, Debs RJ, Desprez PY. Id genes and proteins as promising targets in cancer therapy. Trends in Molecular Medicine. 2004; $10: 387-92$

18. Tsuchiya T, Okaji Y, Tsuno NH, Sakurai D, Tsuchiya N, Kawai K, et al. Targeting Id 1 and Id3 inhibit peritoneal metastasis of gastric cancer. Cancer Science. 2005; 96:784-790.

19. Hiebert SW, Packam G, Strom DK, Haffner R, Oren M, Zambetti G, et al. E2F-1: DP-1 induces p53 and overrides survival factors to trigger apoptosis. Molecular and Cellular Biology. 1995; 11:686468774.

20. Prabhu S, Ignatova A, Park ST, Sun XH. Regulation of expression of the cyclin dependent kinase inhibitor p21 by E2A and Id proteins. Molecular and Cellular Biology. 1997; 17:5888-5896.

21. Chen X, Ko JL, Jayaraman L, Prives C. P53 levels, functional domains, and DNA damage determine the extent of the apoptotic response of tumour cells. Genes and Development. 1996; 11:2438 2451.

22. Florio M, Hernandez MC, Yang H, Shu HK, Cleveland JL, Israel MA. Id 2 promotes apoptosis by a novel mechanism independent of dimerisation to basic helix-loop-helix factors. Molecular and Cellular Biology. 1998; 18:2371-2381.

23. Norton JD, Atherton GT. Coupling of cell growth control and apoptosis functions of ID proteins. Molecular and Cellular Biology. 1998; 18:2371-2381.

24. Yokota Y. Id and development. Oncogene. 2001; 20:8290-8298.

\section{How to cite this article:}

Kyei F, Asante DB, Edekor JAM, Sarpong E, Gavor E, Konja D. Down-regulation of Id1 and Id3 genes affects growth and survival of Human Umbilical Vein Endothelial Cells (HUVECs). J App Biol Biotech. 2016; 4 (02): 023-029. DOI: 10.7324/JABB.2016.40204 